\title{
4-1BB/4-1BBL Interaction Promotes Obesity-Induced Adipose Inflammation by Triggering Bidirectional Inflammatory Signaling in Adipocytes/Macrophages
}

\author{
Thai Hien Tu, ${ }^{1}$ Chu-Sook Kim, ${ }^{1}$ Tsuyoshi Goto, ${ }^{2}$ Teruo Kawada, ${ }^{2}$ \\ Byung-Sam Kim, ${ }^{3}$ and Rina $\mathbf{Y u}^{1}$ \\ ${ }^{1}$ Department of Food Science and Nutrition, University of Ulsan, Ulsan 680-749, Republic of Korea \\ ${ }^{2}$ Graduate School of Agriculture, Kyoto University, Uji, Kyoto 611-0011, Japan \\ ${ }^{3}$ Department of Biological Science, University of Ulsan, Ulsan 689-749, Republic of Korea
}

Correspondence should be addressed to Rina Yu, rinayu@ulsan.ac.kr

Received 14 August 2012; Revised 10 November 2012; Accepted 12 November 2012

Academic Editor: Fábio Santos Lira

Copyright (C) 2012 Thai Hien Tu et al. This is an open access article distributed under the Creative Commons Attribution License, which permits unrestricted use, distribution, and reproduction in any medium, provided the original work is properly cited.

Obesity-induced adipose inflammation is characterized by recruitment of macrophages to adipose tissue and release of inflammatory cytokines. 4-1BB, a costimulatory receptor, modulates inflammatory processes through interaction with its ligand $4-1 \mathrm{BBL}$ on immune cell surfaces. In this study, we examined whether a 4-1BB/4-1BBL interaction between adipocytes and macrophages participates in obesity-induced adipose inflammation. We found that 4-1BB was expressed on adipocytes and was upregulated by obesity-related factors, which also enhanced 4-1BBL expression on macrophages. 4-1BB and/or 4-1BBL agonists, respectively, activated inflammatory signaling molecules (MAPK/I $\kappa \mathrm{B} \alpha$ and MAPK/Akt) in adipocytes and macrophages and enhanced the release of inflammatory cytokines (MCP-1, TNF- $\alpha$, and IL-6). Moreover, disruption of the 4-1BB/4-1BBL interaction decreased the release of inflammatory cytokines from contact cocultured adipocytes/macrophages. These findings indicate that 41BB/4-1BBL-mediated bidirectional signaling in adipocytes/macrophages promotes adipose inflammation. 4-1BB and 4-1BBL may be useful targets for protection against obesity-induced adipose inflammation.

\section{Introduction}

Obesity-induced inflammation is considered to be a potential cause of metabolic disorders such as insulin resistance, type 2 diabetes, and cardiovascular diseases [1-3]. Adipose tissue actively participates in obesity-induced inflammation through recruitment of macrophages and $\mathrm{T}$ cells and release of inflammatory cytokines (monocyte chemotactic protein-1, MCP-1; tumor necrosis factor alpha, TNF- $\alpha$; interleukin-6, IL-6) which modulate adipocyte differentiation, metabolism, and local/systemic inflammatory responses, causing undesirable metabolic imbalances $[2,4]$. Interestingly, recent studies have shown that direct contact coculture of adipocytes and macrophages results in markedly elevated release of inflammatory cytokines [5-7], indicating that interaction between cell surface molecules on these cells is important for promoting their inflammatory responses.

4-1BB (also known as CD137 and TNFRSF9) is a classic example of a costimulatory molecule, and a well-known inflammatory receptor that is expressed by activated $\mathrm{T}$ cells at sites of inflammation [8]. Stimulation of 4-1BB on $\mathrm{T}$ cells leads to cell expansion, cytokine production, and development of cytolytic effector functions [9]. 4-1BB ligand (4-1BBL, also known as CD137L and TNFSF9) is highly expressed by most immune and many nonimmune cells and can receive and transmit reverse signals into cells such as macrophages [10]. Accumulating evidence shows that bidirectional cell surface $4-1 \mathrm{BB} / 4-1 \mathrm{BBL}$ interactions in immune cells are critical in initiating and modulating 
various inflammatory responses (e.g., rheumatoid arthritis, autoimmune myocarditis, and hematological malignancies) [11-13]. Moreover, 4-1BB/4-1BBL-mediated interactions also occur between immune and nonimmune cells, again influencing inflammatory responses. For example, interaction between 4-1BB and 4-1BBL on endothelial cells and macrophages is involved in vascular inflammation $[14,15]$, and interaction between the two molecules on epithelial cells and natural killer cells is involved in renal ischemiareperfusion injury [16]. We previously showed that expression of $4-1 \mathrm{BB}$ and $4-1 \mathrm{BBL}$ was upregulated in adipose tissue that was inflamed due to obesity, and that ablation of $4-1 \mathrm{BB}$ reduced adipose inflammation [17]. Hence, we hypothesized that interaction between $4-1 \mathrm{BB}$ and $4-1 \mathrm{BBL}$ on adipose cells and immune cells such as macrophages plays a role in adipose inflammation in obesity.

In this study, we show for the first time that $4-1 \mathrm{BB}$ is expressed on adipocytes and is upregulated by obesityrelated factors, and we demonstrate that $4-1 \mathrm{BB} / 4-1 \mathrm{BBL}-$ mediated bidirectional signaling in adipocytes/macrophages plays a crucial role in initiating and promoting the obesityinduced adipose inflammatory cascade.

\section{Materials and Methods}

2.1. Animals. C57BL/6 mice (male, 8 weeks old) (Orient Ltd., Busan, Korea) were fed a high-fat diet (HFD, 60\% of calories from fat (Research Diets Inc., New Brunswick, NJ, USA); obese mice) or a low fat diet (LFD; $10 \%$ of calories from fat (Research Diets); nonobese mice) for 9 weeks. All animal experiments were approved by the animal ethics committee of the University of Ulsan and conformed to National Institutes of Health guidelines.

2.2. Antibodies. Nude mice were primed with pristane and injected intraperitoneally with a subcloned hybridoma producing an agonistic monoclonal antibody ( $\mathrm{Ab}$ ) against 4-1BB (3E1) to induce ascite formation [18]. The monoclonal $\mathrm{Ab}$ was purified from the ascites fluid by affinity column chromatography with protein G-Sepharose (SigmaAldrich). Recombinant 4-1BB Fc (r4-1BB Fc) was purchased from Adipogen (Seoul, Korea). Antagonistic monoclonal Ab against 4-1BBL (TKS-1) was purchased from e-Bioscience (San Diego, CA, USA). Rat immunoglobulin G (Rat IgG) and human IgG1 were purchased from Sigma-Aldrich (St. Louis, MO, USA) and used as control.

2.3. Cell Cultures and Treatments. The murine macrophage cell line Raw264.7 was obtained from the Korean Cell Line Bank (KCLB40071, Seoul, Korea). This cell line was maintained in RPMI1640 (Gibco BRL, NY, USA) containing $10 \%(\mathrm{vol} / \mathrm{vol}$ ) FBS (fetal bovine serum) (Gibco $\mathrm{BRL}, \mathrm{NY}, \mathrm{USA}$ ) and incubated at $37^{\circ} \mathrm{C}$ in humidified $5 \%$ $\mathrm{CO}_{2}$. 3T3-L1 preadipocytes were maintained in DMEM (Dulbecco's Modified Eagle Medium) high glucose (Gibco BRL, NY, USA) containing 10\% FBS. Confluent 3T3-L1 preadipocytes (day 0) were incubated in DMEM containing
$10 \mu \mathrm{g} / \mathrm{mL}$ insulin (Sigma-Aldrich), 0.25 $\mu \mathrm{M}$ DEX (dexamethasone, Sigma-Aldrich), 0.5 mM IBMX (3-isobutyl1-methyl-xanthine, Sigma-Aldrich), and 10\% FBS for 2 days. Briefly, 3T3-L1 cells were differentiated into mature adipocytes by incubation in DMEM with 10\% FBS and $5 \mu \mathrm{g} / \mathrm{mL}$ insulin for 2 days. Mature adipocytes were maintained in this medium, and the culture medium was replaced with fresh medium every 2 days. Free fatty acid (FFA, palmitic acid mixture, Sigma-Aldrich) was dissolved in ethanol containing bovine serum albumin (BSA, $25 \mu \mathrm{M}$ ) and conjugated with BSA at a 10:1 molar ratio before use. 3T3L1 adipocytes $\left(3 \times 10^{5}\right.$ cells/well $)$ or Raw264.7 macrophages $\left(3 \times 10^{5}\right.$ cells/well $)$ in 24 -well plates, were treated with obesity-related factors (palmitic acid:pal, lipopolysaccharide: LPS) for $24 \mathrm{~h}$ or $4 \mathrm{~h}$, respectively. To stimulate $4-1 \mathrm{BB}$ on adipocytes, 3T3-L1 adipocytes on 24-well plates were incubated with agonistic $4-1 \mathrm{BB} \mathrm{Ab}(3 \mathrm{E} 1,1 \mu \mathrm{g} / \mathrm{mL})$ or rat IgG for $48 \mathrm{~h}$ in serum-free medium. To immobilize r4-1BB Fc or human IgG1 on culture plates, r4-1BB Fc or human IgG1 were incubated in 24 -well plates at $37^{\circ} \mathrm{C}$ for $1 \mathrm{~h}$ in a $\mathrm{CO}_{2}$ incubator and the wells were rinsed with phosphate buffered saline (PBS). The plates were then incubated with RPMI $(10 \% \mathrm{FBS})$ at $37^{\circ} \mathrm{C}$ for $1 \mathrm{~h}$ in a $\mathrm{CO}_{2}$ incubator and the wells were rinsed with PBS. Raw264.7 macrophages were incubated at $5 \times 10^{5}$ cells/well in 24-well flat-bottomed plates precoated with $100 \mathrm{ng} / \mathrm{mL}$ r4-1BB Fc or human IgG1 for $24 \mathrm{~h}$.

2.4. Isolation of Stromal Vascular Cells from Adipose Tissue. To isolate the stromal vascular fraction (SVF) of adipose tissue, epididymial fat pads of C57BL/6 mice (male, 8 weeks old) were minced and digested for 30 minutes at $37^{\circ} \mathrm{C}$ with type 2 collagenase ( $1 \mathrm{mg} / \mathrm{mL}$; Sigma-Aldrich) in DMEM ( $\mathrm{pH}$ 7.4). The resulting suspensions were centrifuged at $500 \mathrm{~g}$ for 5 minutes. The pellets were resuspended in erythrocyte lysis buffer, and the suspensions incubated at room temperature for 3 minutes, then centrifuged at $500 \mathrm{~g}$ for 5 minutes. After washing in DMEM, the suspensions were passed through sterile $100 \mu \mathrm{m}$ nylon meshes (SPL Lifescience, Pocheon, Korea). The filtrated cells were transferred to $100 \mathrm{~mm}^{2}$ dishes contained DMEM supplemented with $10 \%$ FBS and $0.4 \%$ Fungizone and maintained in an incubator at $37^{\circ} \mathrm{C}$ in $5 \%$ $\mathrm{CO}_{2}$. Cells were allowed to attach and floating cells were removed by aspiration and the culture media was replenished for every day. The SVF cells were collected after 2 day. The SVF cells were plated at $5 \times 10^{5}$ cell/well in 24well plates. The confluent SVF-derived preadipocytes were differentiated into adipocytes by treatment with DMEM containing $10 \mu \mathrm{g} / \mathrm{mL}$ insulin (Sigma-Aldrich), 0.25 $\mu \mathrm{M}$ DEX (Sigma-Aldrich), $0.5 \mathrm{mM}$ IBMX (Sigma-Aldrich), and 10\% FBS for 2 days. Mature adipocytes were maintained in the culture medium that was replaced with fresh medium every 2 days. To detect 4-1BB and 4-1BBL expression on SVF-derived adipocytes exposed to obese factors, these cells incubated with palmitic acid $250 \mu \mathrm{M}$, LPS $100 \mathrm{ng} / \mathrm{mL}$ for $24 \mathrm{~h}$.

2.5. Isolation of Peritoneal Macrophages. C57BL/6 mice (male, 8 weeks old) were intraperitoneally injected with $3 \mathrm{ml}$ 
TABLE 1: Sequences of mouse primers used for qRT-PCR analysis.

\begin{tabular}{lll}
\hline Gene & Forward primer sequence & Reverse primer sequence \\
\hline $4-1 \mathrm{BB}$ & CTCTGTGCTCAAATGGATCAGGAA & TGTGGACATCGGCAGCTACAA \\
$4-1 \mathrm{BBL}$ & CCTGTGTTCGCCAAGCTACTG & CGGGACTGTCTACCACCAACTC \\
MCP-1 & GCATCCACGTGTTGGCTCA & CTCCAGCCTACTCATTGGGATCA \\
TNF- $\alpha$ & AAGCCTGTAGCCCACGTCGTA & GGCACCACTAGTTGGTTGTCTTTG \\
IL-6 & CCACTTCACAAGTCGGAGGCTTA 4 & GCAAGTGCATCATCGTTGTTCATAC \\
$\beta$-actin & TGTGTGTCTGCAGATCGGGTAC & CTTTGGCGGGATTAGTCGAAG \\
\hline
\end{tabular}

of 3\% thioglycollate broth (Difco, Detroit, MI, USA) 4 days before being killed. Peritoneal macrophages were collected by centrifugation in MEM media (Minimum Essential Medium, Gibco) and the resulting pellet was washed and resuspended in culture medium MEM with 10\% FBS. The peritoneal macrophages were purified by adherence to tissue culture plates for 2 hours [19]. To detect 4-1BB and 4-1BBL expression on peritoneal macrophages exposed to obese factors, these cells incubated with palmitic acid $250 \mu \mathrm{M}$, LPS $100 \mathrm{ng} / \mathrm{mL}$ for $4 \mathrm{~h}$.

2.6. Coculture of Adipocytes and Macrophages. 3T3-L1 adipocytes were cultured and differentiated for 6 days. Coculture of adipocytes and macrophages was performed by two methods: direct contact coculture and transwell coculture. In the direct contact system, Raw264.7 macrophages $\left(3 \times 10^{5}\right.$ cells: $50 \%$ macrophages, $3 \times 10^{4}$ cells: $10 \%$ macrophages) or peritoneal macrophages $\left(3 \times 10^{5}\right.$ cells $)$ were placed in 24-well plates containing 3T3-L1 adipocytes $\left(3 \times 10^{5}\right.$ cells $)$. The cells were cultured for $24 \mathrm{~h}$ in contact with each other and harvested. As a control, adipocytes and macrophages were also cultured separately, with cell numbers per well equal to those in the contact system and mixed after harvest. In the trans-well system, cells were cocultured using transwell inserts with a $0.4 \mu \mathrm{m}$ porous membrane (Corning, NY, USA) to separate adipocytes $\left(3 \times 10^{5}\right.$ cells, lower well) from macrophages $\left(3 \times 10^{5}\right.$ cells, upper well). After incubation for $4 \mathrm{~h}, 8 \mathrm{~h}$, and $12 \mathrm{~h}$, the supernatants were harvested.

2.7. Measurement of Cytokine Levels. Cytokine levels in culture supernatants were measured using enzyme-linked immunosorbent assays (ELISA). The assays were conducted using OptEIA mouse TNF $\alpha$, a mouse MCP-1 set (BD Bioscience Pharmingen, CA, USA) and a mouse IL-6 and adiponectin set (R\&D Systems, Minneapolis, MN, USA), and IL-10 kit (R\&D Systems, Minneapolis, MN, USA). Values for cytokine levels were derived from standard curves using the curve-fitting program SOFTmax (Molecular Devices, Sunnyvale, CA, USA).

2.8. Quantitative Real-Time PCR ( $q R T-P C R)$. Total RNA extracted from cultured cells was reverse transcribed to generate cDNA using M-MLV reverse transcriptase (Promega, Madison, WI, USA). Real-time PCR amplification of the cDNA was performed in duplicate with a SYBR premix Ex
Taq kit (TaKaRa Bio Inc., Foster, CA, USA) using a Thermal Cycler Dice (TaKaRa Bio Inc., Japan). All reactions were performed by the same procedure: initial denaturation at $95^{\circ} \mathrm{C}$ for $10 \mathrm{~s}$, followed by 45 cycles of $95^{\circ} \mathrm{C}$ for $5 \mathrm{~s}$ and $60^{\circ} \mathrm{C}$ for $30 \mathrm{~s}$. All values for genes of interest were normalized to values for housekeeping genes (36B4 for adipocytes; $\beta$-actin for macrophages and cocultures). Mouse primer sequences used are shown in Table 1.

Data were analyzed using Thermal Cycler Dice Real Time System Software (Takara Bio, Inc.). Relative standard curves were generated by plotting the cycle threshold $(\mathrm{Ct})$ values. Base on the Ct values obtained from each sample, the relative amounts of target genes were calculated using the standards curves with software provided by Takara Thermal Cycler Dice Real Time System.

2.9. Separation of Adipocytes and Macrophages. Cocultured 3T3-L1 adipocytes and Raw264.7 macrophages in equal numbers (as described earlier) were separated following the manufacturer's protocol, using the CD11b MicroBeads system (MACS; Miltenyi Biotec, Sunnyvale, CA, USA). Briefly, cocultured cells were collected, washed twice with buffer (PBS supplemented with $2 \mathrm{mM}$ EDTA and $0.5 \%$ bovine serum albumin-BSA), and incubated with CD11b microbeads for $15 \mathrm{~min}$ at $4^{\circ} \mathrm{C}$. Washed and resuspended cells were applied to MACS column, which retained CD11b ${ }^{+}$ cells and allowed negative cells (adipocytes) to pass through. The column was then removed from the separator and placed on a suitable collection tube. Appropriate amounts of column buffer were pipetted onto the column to flush out positive cells (macrophages) using a plunger supplier with the column. This method resulted in 90\% to 95\% pure

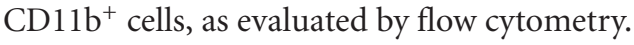

2.10. Flow Cytometry (FACS) Analysis. 3T3-L1 adipocytes and Raw264.7 macrophages treated with obesity-related factors (as described earlier) were gently trypsinized, washed twice in PBS, and incubated with Fcy receptor-blocking antibodies (24G2) for 10 minutes on ice, then stained with phycoerythrin (PE) conjugated anti-4-1BB (eBioscience, San Diego, CA, USA), anti-4-1BBL (eBioscience), or anti-Rat IgG2 ${ }_{\mathrm{a}, \mathrm{k}}$ (eBioscience), Golden Syrian Hamster IgG (eBioscience) and anti-CD11b (eBioscience) as a control to define the gate for adipocytes/macrophages. The cells were then washed with FACS buffer and analyzed on a FACSCalibur (BD Biosciences, San Jose, CA, USA) with CellQuest software 


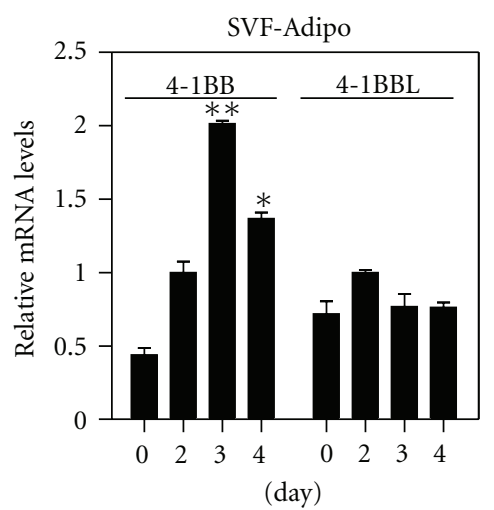

(a)

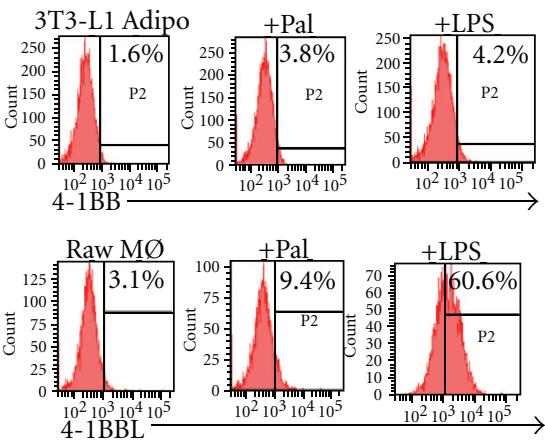

(d)

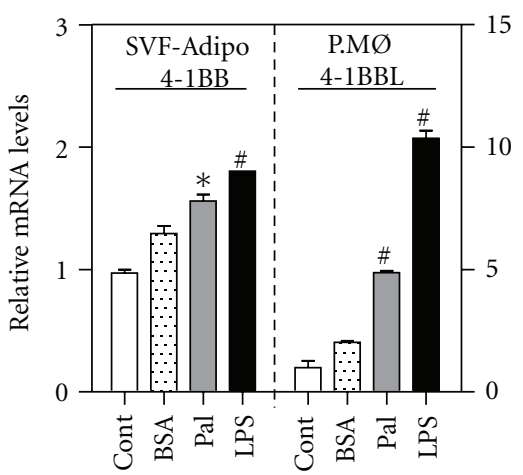

(b)

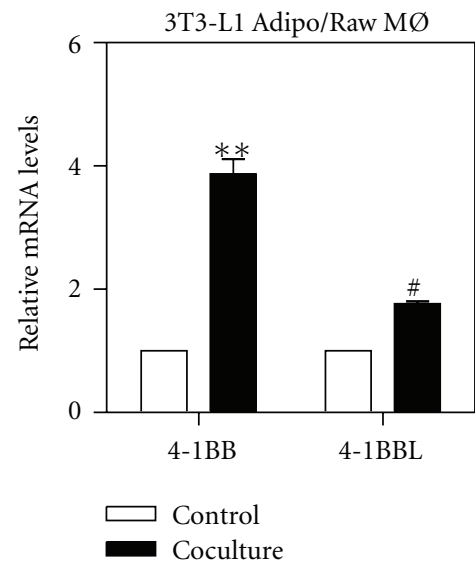

(e)

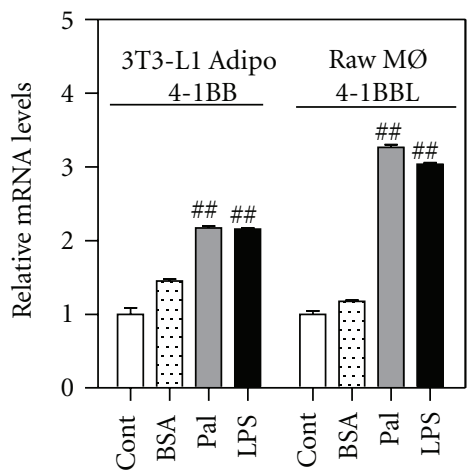

(c)

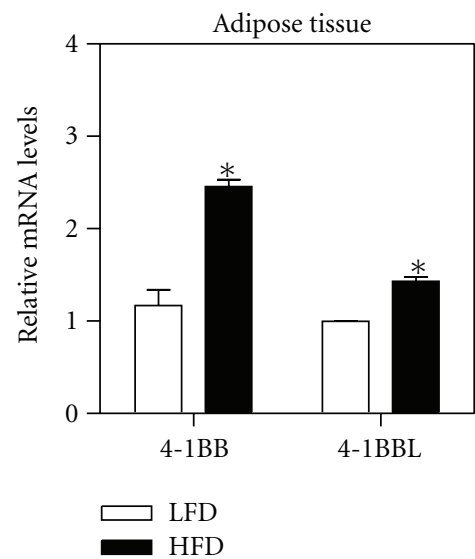

(f)

FIGURE 1: 4-1BB and 4-1BBL expression is upregulated by obesity-related factors in adipocytes and macrophages. 4-1BB and 4-1BBL mRNA expression in SVF-derived adipocytes (a) during adipogenesis. SVF-derived confluent preadipocytes (day 0) were differentiated into adipocytes (days 2-4), as described in Section 2. 4-1BB and 4-1BBL mRNA levels in SVF-derived adipocytes and peritoneal macrophages treated with obesity-related factor $(250 \mu \mathrm{M}$ Pal, $100 \mathrm{ng} / \mathrm{mL}$ LPS) for 24 and $4 \mathrm{~h}$ (b). 4-1BB/4-1BBL mRNA (c) and protein expression (d) in 3T3-L1 adipocytes and Raw264.7 macrophages treated with obesity-related factor $(250 \mu \mathrm{M} \mathrm{Pal}, 100 \mathrm{ng} / \mathrm{mL} \mathrm{LPS})$ for $24 \mathrm{~h}$ and $4 \mathrm{~h}$. mRNA was measured by qRT-PCR, and protein levels were detected by FACS. 4-1BB and 4-1BBL mRNA levels in 3T3-L1 adipocytes/Raw264.7 macrophages cocultured for $24 \mathrm{~h}(\mathrm{e})$, control indicates mixed adipocytes/macrophages, which were cultured separately for $24 \mathrm{~h}$ and mixed after harvest, and in the epididymal adipose tissue (f) of mice fed a high-fat diet (HFD) or low-fat diet (LFD) ( $n=4$ mice per group). Levels of mRNA were estimated by qRT-PCR. Pal, palmitic acid; Adipo, adipocytes; P.M $\varnothing$, peritoneal macrophages; Raw M $\varnothing$, Raw264.7 macrophages. Data are the mean \pm SEM of three independent experiments performed in duplicate. ${ }^{*} P<0.05 ;{ }^{* *} P<0.01$; ${ }^{*} P<0.005$; $\# P<0.001$ (compared with control).

(BD Biosciences). Number in the graphs indicates the percentages of positive cells.

2.11. Western Blot Analysis. 3T3-L1 adipocytes were plated at $1 \times 10^{6}$ cells/well in 6 -well plates and incubated with 3E1 $(1 \mu \mathrm{g} / \mathrm{mL})$ or rat IgG for $3 \mathrm{~h}$. Raw264.7 macrophages were plated at $1 \times 10^{6}$ cells/well in 6-well plates coated with $\mathrm{r} 4$ $1 \mathrm{BB}$ Fc or human IgG for $1 \mathrm{~h}$. The 3E1-treated adipocytes and $\mathrm{r} 4-1 \mathrm{BB}$ Fc-treated macrophages were rinsed with PBS, resuspended by scraping in lysis buffer $(10 \mathrm{mM}$ Tris- $\mathrm{HCl}$, $10 \mathrm{mM} \mathrm{NaCl}, 0.1 \mathrm{mM}$ EDTA, $50 \mathrm{mM} \mathrm{NaF}, 10 \mathrm{mM} \mathrm{Na}_{4} \mathrm{P}_{2} \mathrm{O}_{7}$, $1 \mathrm{mM} \mathrm{MgCl} 2,0.5 \%$ deoxycholate, $1 \%$ IGEPAL, and protease inhibitors cocktail), and centrifuged at $3000 \mathrm{rpm}$ for 5 minutes. Samples containing $10-30 \mu \mathrm{g}$ of total protein were subjected to western blot analysis using polyclonal antibodies to phosphorylated IKK (I kappa B kinase alpha/beta; p-IKK $\alpha / \beta$, Ser180/Ser181), total IKK $\beta$, p-p38 MAPK (mitogen-activated-protein kinase), p-JNK (c-Jun amino-terminal kinase), total JNK, p-Akt (protein kinase B; Ser473), total Akt (Cell Signaling, Danvers, MA, USA), and $\mathrm{I} \kappa \mathrm{B} \alpha$ (inhibitor of nuclear factor- $\kappa \mathrm{B}$ alpha; Santa Cruz Biotechnology, Santa Cruz, CA, USA), and $\beta$-actin (Sigma).

2.12. Statistical Analysis. Results are presented as means \pm SEM of three independent performed in duplicate. Statistical comparisons were performed using Student's $t$-test or ANOVA with Duncan's multiple-range test. Differences were considered to be significant at $P<0.05$. 


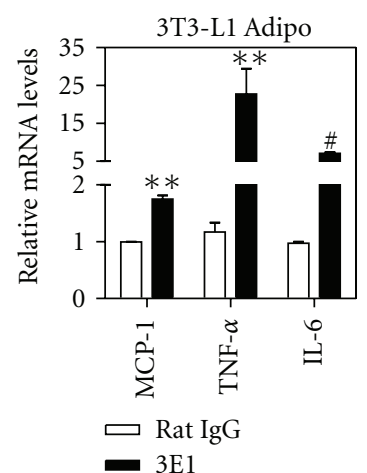

(a)

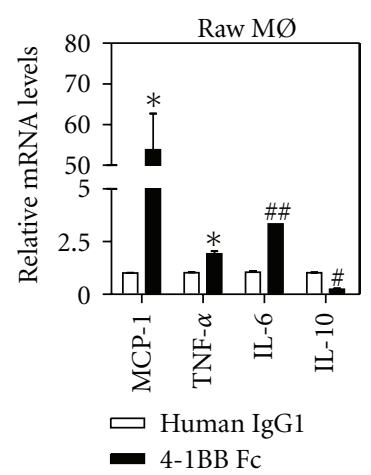

(c)

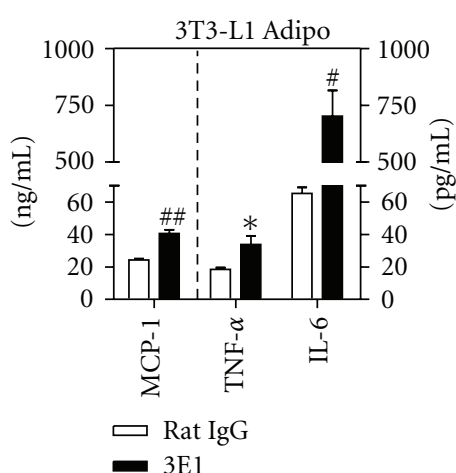

(b)

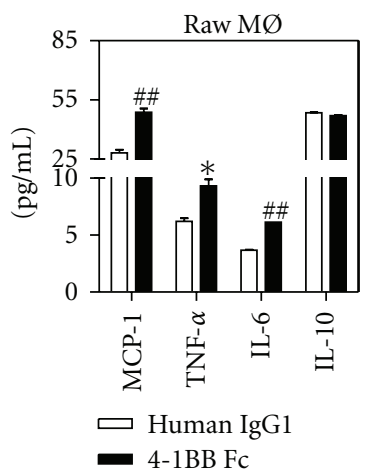

(d)
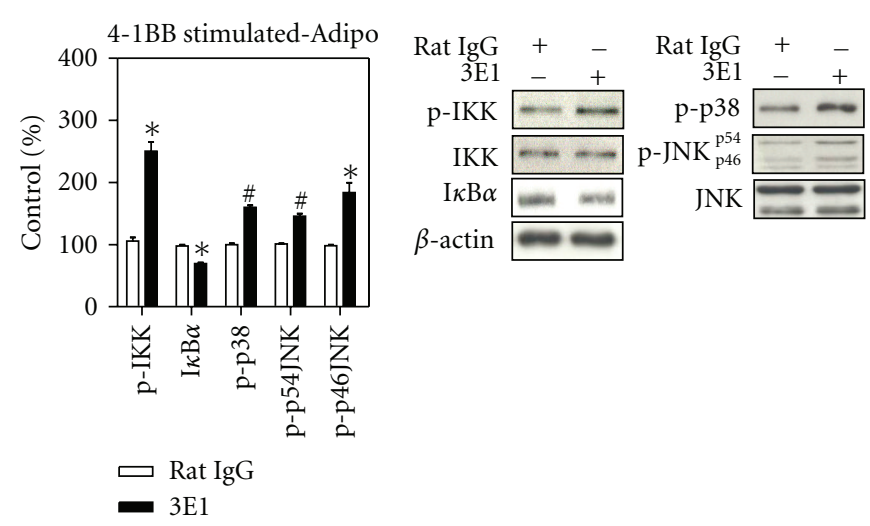

(e)

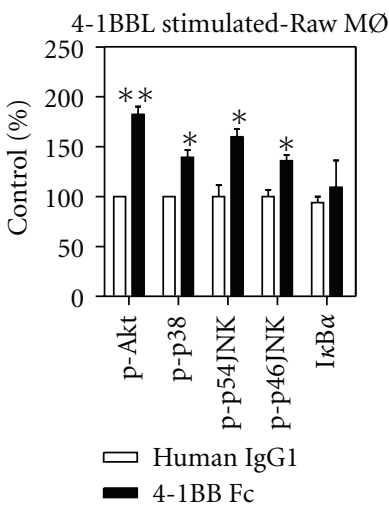

$\mathrm{H}-\operatorname{IgG} 1+-$

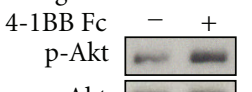

Akt

$\mathrm{I} \kappa \mathrm{B} \alpha$

$\beta$-actin
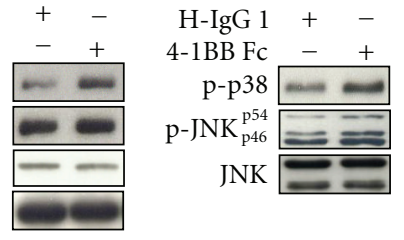

(f)

FIGURE 2: 4-1BB or 4-1BBL stimulation enhances release of various inflammatory cytokines from adipocytes or macrophages. 3T3-L1 adipocytes and Raw264.7 macrophages were treated with $1 \mu \mathrm{g} / \mathrm{mL} 3 \mathrm{E} 1$ and $100 \mathrm{ng} / \mathrm{mL} \mathrm{r4-1BB} \mathrm{Fc,} \mathrm{respectively,} \mathrm{for} 48-24 \mathrm{~h}$. MCP-1, TNF$\alpha$, IL-6, and IL-10 transcripts and proteins were then measured in the 3T3-L1 adipocytes (a-b) and Raw264.7 macrophages (c-d). The phosphorylation of IKK $\alpha / \beta(\mathrm{p}-\mathrm{IKK} \alpha / \beta) / \mathrm{IKK} \beta, \mathrm{I} \kappa \mathrm{B} \alpha$, p-p38, p-JNK/JNK, pAkt/Akt and $\beta$-actin were measured by western blotting. 3T3$\mathrm{L} 1$ adipocytes were incubated with $1 \mu \mathrm{g} / \mathrm{mL} 3 \mathrm{E} 1 \mathrm{for} 3 \mathrm{~h}(\mathrm{e})$, and Raw264.7 macrophages with $100 \mathrm{ng} / \mathrm{mL} \mathrm{r} 4-1 \mathrm{BB}$ Fc for $1 \mathrm{~h}$ (f). Results of densitometry were showed as percentage of control. Data are the mean \pm SEM of three independent experiments performed in duplicate. ${ }^{*} P<0.05 ;{ }^{* *} P<0.01 ;{ }^{\#} P<0.005 ;{ }^{\# \#} P<0.001$ (compared with control).

\section{Results}

3.1. Expression of $4-1 B B$ and $4-1 B B L$ in Adipocytes/Macrophages and Adipose Tissue. We first measured expression of $4-1 \mathrm{BB}$ during adipogenesis at the mRNA level using qRT-PCR. We found that levels of 4-1BB transcripts were greater in SVF-derived adipocytes after differentiation (Figure 1(a)). Importantly, obesity-related substances such as palmitic acid and LPS significantly upregulated levels of 4-1BB transcripts in SVF-derived adipocytes and 41BBL transcripts in peritoneal macrophages (Figure 1(b)). The upregulation of the transcripts is confirmed in 3T3L1 adipocytes and/or Raw264.7 macrophages (Figure 1(c)). FACS analysis also revealed that 4-1BB protein on 3T3-L1 adipocytes and 4-1BBL protein on Raw264.7 macrophages (Figure 1(d)) were increased by these obesity-related factors. In addition, $4-1 \mathrm{BB}$ and $4-1 \mathrm{BBL}$ transcripts increased in cocultured adipocytes/macrophages (Figure 1(e)), as well as in the epididymal adipose tissue of obese mice fed an HFD (Figure 1(f)).
3.2. Release of Inflammatory Cytokines and Activation of Inflammatory Signaling Molecules by 4-1BB and 4-1BBL Stimulation in Adipocytes and Macrophages, Respectively. To examine whether 4-1BB on adipocytes or 4-1BBL on macrophages provide an inflammatory signal, we treated each cell type with agonists that specifically stimulate these molecules; 3T3-L1 adipocytes were treated with an agonistic 4-1BB antibody (3E1) for $48 \mathrm{~h}$, and Raw264.7 macrophages with $\mathrm{r} 4-1 \mathrm{BB}-\mathrm{Fc}$ for $24 \mathrm{~h}$ and we then measured levels of inflammatory cytokines in the respective cells. Both 41BB stimulation of adipocytes and 4-1BBL stimulation of macrophages markedly increased the production of proinflammatory cytokines such as MCP-1, TNF- $\alpha$, and IL- 6 at the mRNA (Figures 2(a), and 2(c)) and protein levels (Figures 2(b) and 2(d)). Adiponectin secretion from adipocytes was not altered by 4-1BB stimulation (data not shown). 4-1BBL stimulation of macrophages decreased the transcripts of IL-10 (Figure 2(c)), but no change was observed in IL-10 protein release (Figure $2(\mathrm{~d})$ ). 


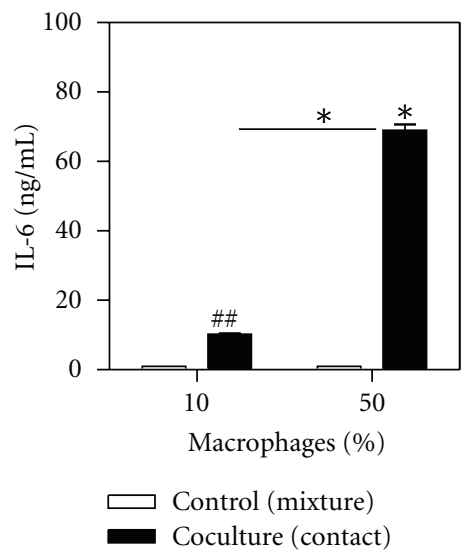

(a)

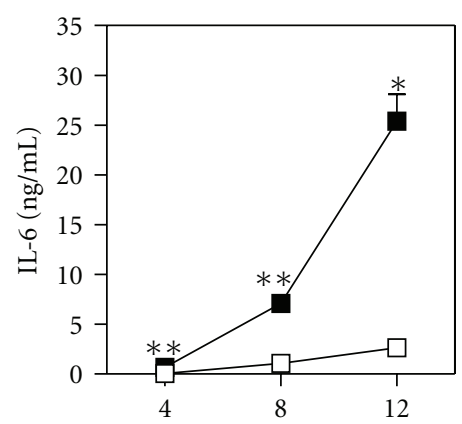

(h)

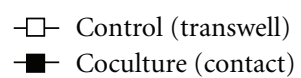

(d)

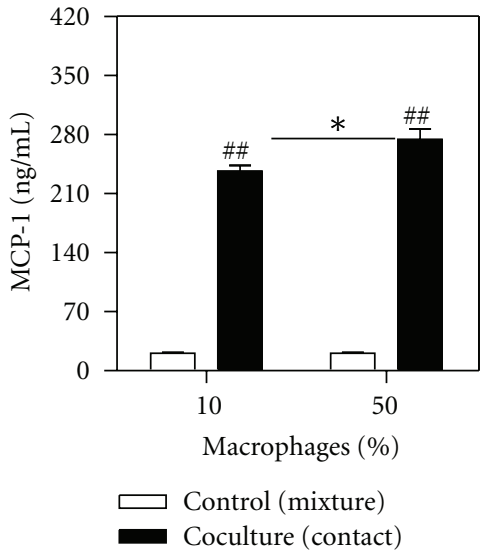

(b)

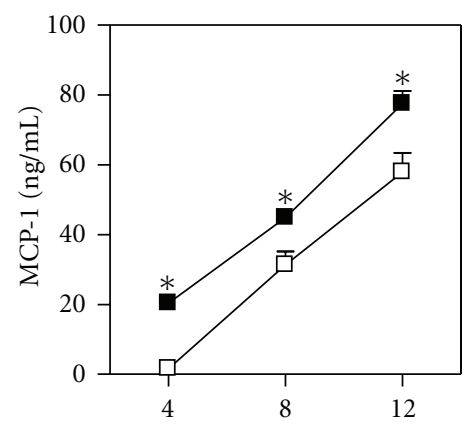

(h)

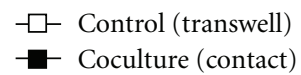

(e)

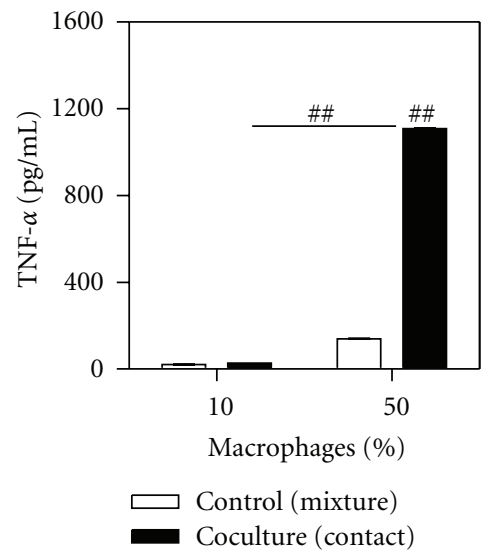

(c)

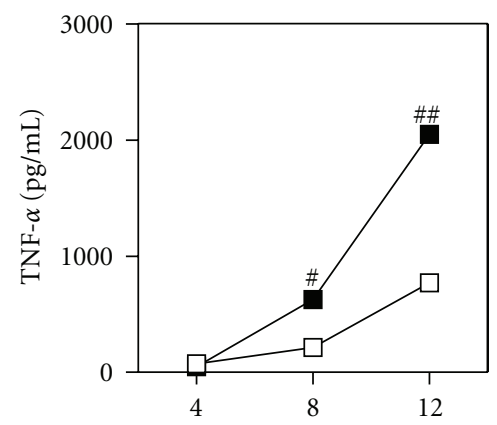

(h)

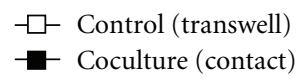

(f)

FIGURE 3: Release of inflammatory cytokines is enhanced in cocultures of 3T3-L1 adipocytes and Raw264.7 macrophages. Release of cytokines (IL-6, MCP-1, and TNF- $\alpha$ ) from 3T3-L1 adipocytes cocultured with Raw264.7 macrophages (10\% or 50\%) for 24h (a-c). Control indicates mixed adipocytes/macrophages, which were cultured separately for $24 \mathrm{~h}$ and mixed after harvest. Release of cytokines (IL-6, MCP1 , and TNF- $\alpha$ ) from the contact coculture with 50\% Raw264.7 macrophages or transwell system for indicated times ( $4 \mathrm{~h}, 8 \mathrm{~h}$, and $12 \mathrm{~h})(\mathrm{d}-\mathrm{f})$. Cell-free supernatants were collected, and concentrations of these inflammatory cytokines were determined by ELISA. Data are the mean \pm SEM of three independent experiments performed in duplicate. ${ }^{*} P<0.05 ;{ }^{* *} P<0.01{ }^{\#} P<0.005 ;{ }^{\# \#} P<0.001$ (compared with control).

To understand the molecular mechanisms by which 4-1BB and/or 4-1BBL activate inflammatory signaling in adipocytes and/or macrophages, we examined the effects of $4-1 \mathrm{BB} / 4-1 \mathrm{BBL}$ stimulation on intracellular signaling molecules. Stimulation of 4-1BB on adipocytes increased the phosphorylation of p38 MAPK and JNK as well as the phosphorylation of IKK, the upstream molecule of NF- $\kappa \mathrm{B}$, and induced $\mathrm{I} \kappa \mathrm{B} \alpha$ degradation (Figure $2(\mathrm{e})$ ), while stimulation of 4-1BBL increased phosphorylation of Akt and p38 MAPK, and JNK (Figure 2(f)), but had no effect on degradation of $\mathrm{I} \kappa \mathrm{B} \alpha$ protein (Figure $2(\mathrm{f})$ ) and phosphorylation of IKK (data not shown). Consistent with previous reports [20,21], 4-1BBL signaling not only activated p38 MAPK but also induced Akt activation in macrophages, leading increased inflammatory cytokines expression.

\subsection{Release of Inflammatory Cytokines in a Contact Coculture} System. Because 4-1BB/4-1BBL stimulation enhanced the release of inflammatory cytokines from adipocytes and/or macrophages, respectively, we investigated whether cellcell interaction via surface molecules, presumably $4-1 \mathrm{BB} / 4$ $1 \mathrm{BBL}$, has a role in initiating and triggering inflammatory responses. We first cocultured 3T3-L1 adipocytes and Raw264.7 macrophages in a direct contact system and found that the production of inflammatory cytokines IL-6, MCP-1, and TNF- $\alpha$ was correlated with the number of macrophages in the culture (Figures 3(a)-3(c)) and increased with time (Figures 3(d)-3(f)).

3.4. Effect of Disruption of the Interaction between 4-1BB and 4-1BBL on Release of Inflammatory Cytokines in a Contact Coculture System. To test whether the 4-1BB/4-1BBLmediated interaction between adipocytes and macrophages participates in the inflammatory response in the contact cocultured 3T3-L1 adipocytes/Raw264.7 macrophages, we blocked the interaction using a neutralizing antibody (TKS1). The neutralizing monoclonal antibody reacts specifically with mouse 4-1BBL, by which 4-1BBL cannot bind to 4-1BB 


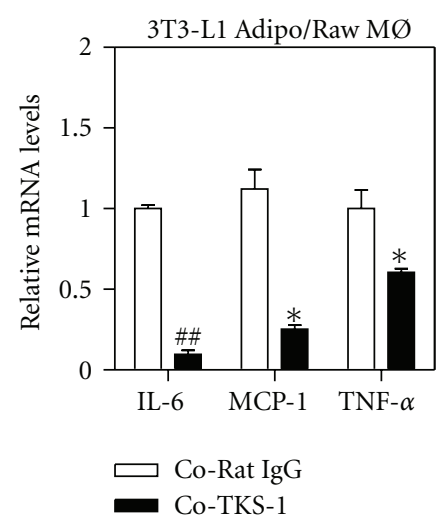

(a)

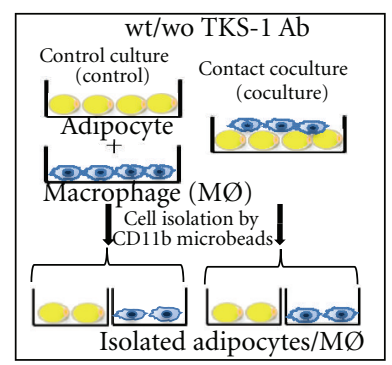

(d)

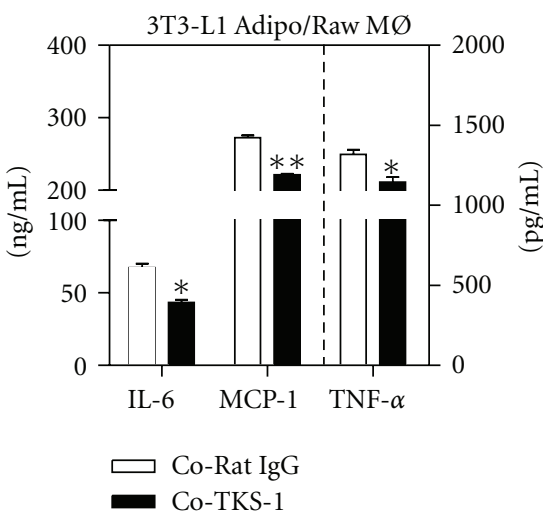

(b)

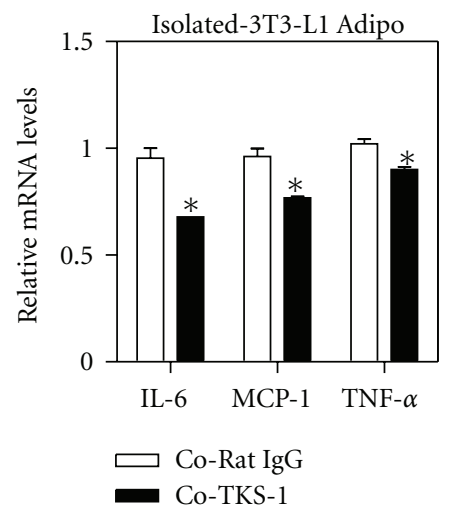

(e)

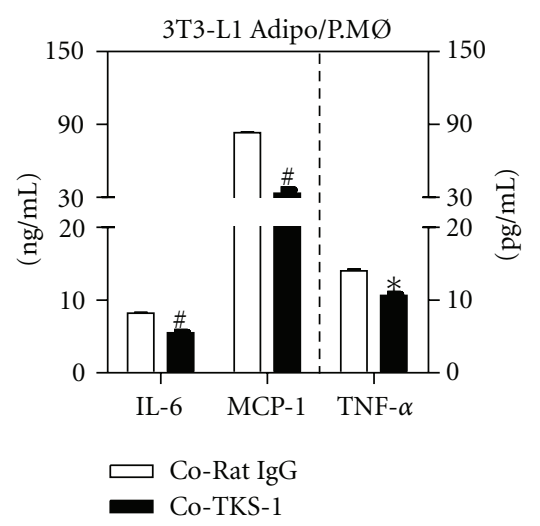

(c)

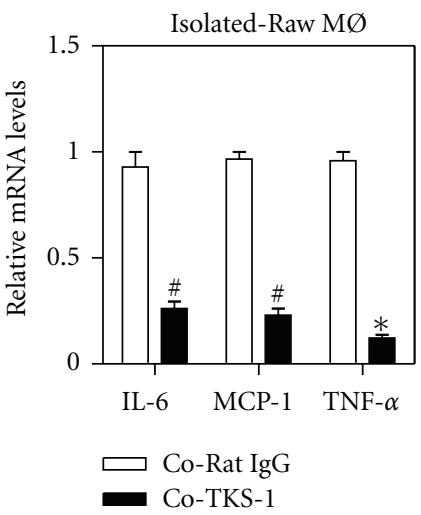

(f)

FIGURE 4: Disruption of the 4-1BB/4-1BBL interaction suppresses expression of inflammatory cytokines in direct contact cocultures. Raw264.7 macrophages were seeded onto 3T3-L1 adipocytes with/without pretreated with neutralizing anti-4-1BBL antibody (TKS-1) or Rat IgG $(5 \mu \mathrm{g} / \mathrm{mL})$ in serum-free medium for $24 \mathrm{~h}$. Total RNAs were isolated and analyzed the level of IL-6, MCP-1, and TNF- $\alpha$ by qRTPCR (a). The protein levels of IL-6, MCP-1, and TNF- $\alpha$ in cell-free supernatants were collected from 3T3-L1 adipocytes and Raw264.7 macrophages coculture (b) and from cocultures of 3T3-L1 adipocytes and peritoneal macrophages (c). Illustration of coculture system with adipocytes/macrophages (d). After $24 \mathrm{~h}$ coculture of Raw264.7 macrophages and 3T3-L1 adipocytes, these cells were separated using the CD11b MicroBead system. Levels of inflammatory cytokine mRNAs were detected in the adipocytes (e) and macrophages (f). Co, coculture. Data are the mean \pm SEM of three independent experiments performed in duplicate. ${ }^{*} P<0.05 ;{ }^{* *} P<0.01 ;{ }^{\#} P<0.005 ;{ }^{\#} P<0.001$ (compared with rat IgG-treated cells).

receptor and can interrupt the interaction between $4-1 \mathrm{BBL}$ and 4-1BB. Hence, both 4-1BB-mediated signal in adipocytes and 4 -1BBL-mediated signal in macrophages can be blunted by TKS-1 treatment. We found that treatment with TKS1 significantly reduced levels of IL-6, MCP- 1 , and TNF- $\alpha$ mRNA in the contact cocultured adipocytes/macrophages (Figure 4(a)). The reduction in the expression of these inflammatory cytokines was confirmed at the protein level (Figure 4(b)). Moreover, we also found that disruption of the interaction between 4-1BB and 4-1BBL reduced the release of inflammatory cytokines from peritoneal macrophages cocultured with adipocytes (Figure 4(c)). To examine the relative contributions of $4-1 \mathrm{BB}$ and $4-1 \mathrm{BB}$ signaling to inflammatory gene expression in the cocultured adipocytes/macrophages, we separated the macrophages from the adipocytes and measured levels of inflammatory cytokine transcripts in the two types of cell (Figure 4(d)). The neutralizing antibody significantly reduced the increase in levels of IL-6, MCP1 , and TNF- $\alpha$ mRNAs in the adipocytes as well as in the macrophages (Figures 4(e) and 4(f)).

\section{Discussion}

Obesity-induced adipose inflammation is characterized by recruitment of macrophages into adipose tissue, and the macrophages are an important source of inflammatory responses. Cell-cell contact between adipocytes and macrophages is considered to be important for triggering inflammatory pathways in adipose tissue $[5,6]$, although it is unclear which molecules are involved. Recent studies have shown that the engagement of co-stimulatory receptor $4-1 \mathrm{BB}$ with its ligand 4-1BBL through cell-cell contact modulates various inflammatory responses $[13,14$, 16]. In previous work, we found that $4-1 \mathrm{BB}$ deficiency 


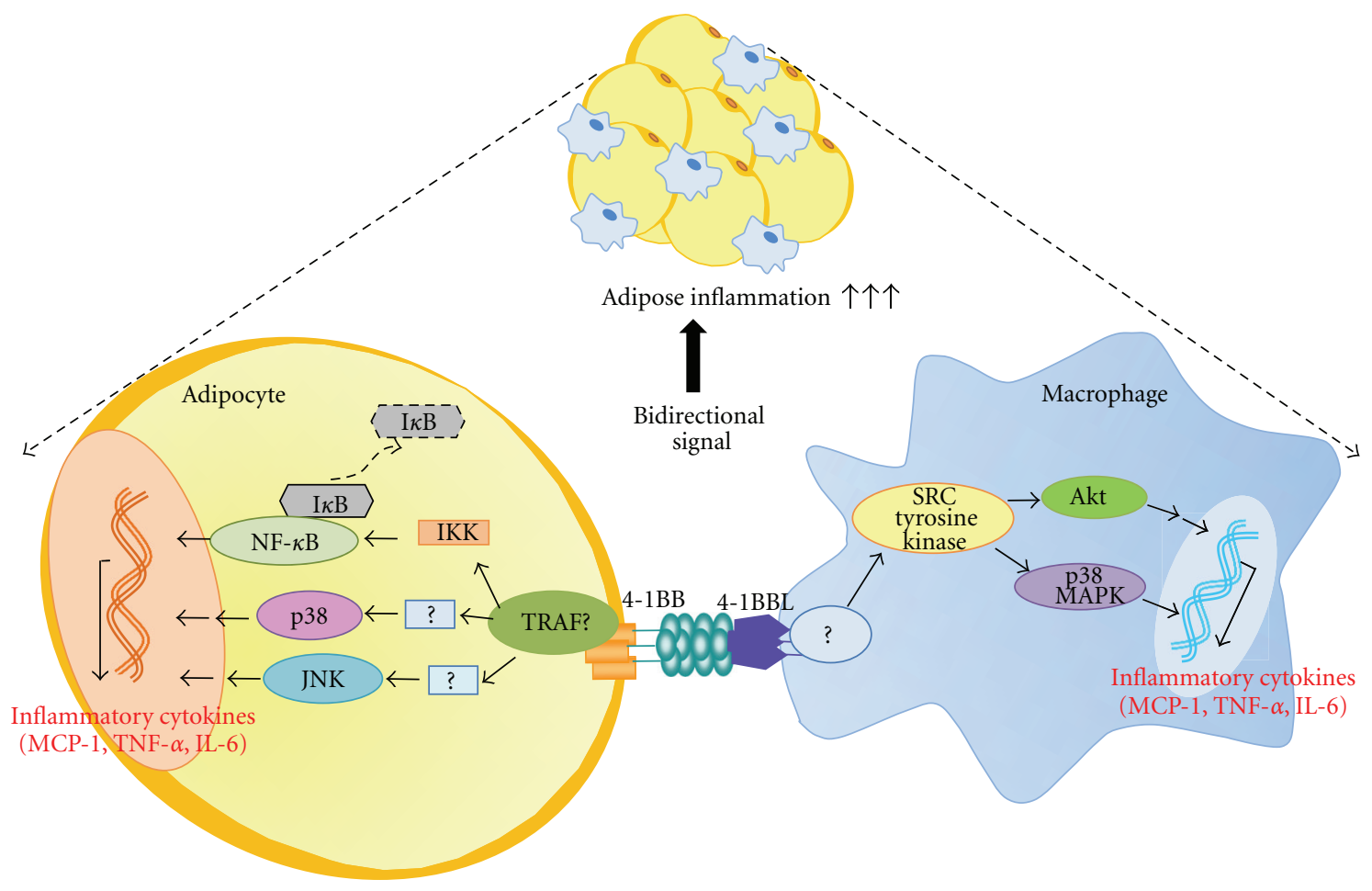

FIGURE 5: Schematic representation of the bidirectional signal transduction induced by 4-1BB/4-1BBL-mediated interaction between adipocytes and macrophages. The release of inflammatory cytokines (MCP-1, TNF- $\alpha$, and IL-6) in response to the bidirectional signaling appears to participate in adipose inflammation.

reduced adipose inflammation by decreasing macrophage recruitment and the release of inflammatory cytokines [17]. Based on these findings, we hypothesized that 41BB/4-1BBL-mediated cell-cell interaction between adipose cells and macrophages might be important in the onset and/or maintenance of obesity-induced adipose inflammation. Interestingly, 4-1BB transcripts in adipocytes and 4$1 \mathrm{BBL}$ transcripts in macrophages were markedly upregulated by obesity-related factors (e.g., FFA and LPS), and their expression was also strongly increased in contact cocultured adipocytes/macrophages. Moreover, the upregulation of these molecules was accompanied by enhanced release of inflammatory cytokines from the cells. These findings together with the upregulation in obese adipose tissue and the reduction of adipose inflammation in 4-1BB-deficient obese mice [17] suggest that 4-1BB and 4-1BBL participate in the onset and/or promotion of adipocytes/macrophageinduced inflammatory responses.

In order to see whether 4-1BB on adipocytes or 4$1 \mathrm{BBL}$ on macrophages was responsible for the inflammatory signals that triggered inflammatory responses, we stimulated the cells with agonists which bind specifically to either $4-1 \mathrm{BB}$ or $4-1 \mathrm{BBL}$. We found, for the first time, that stimulation of $4-1 \mathrm{BB}$ on adipocytes markedly increased the release of inflammatory cytokines MCP-1, TNF- $\alpha$, and IL6. Stimulation of 4-1BBL-mediated reverse signaling, which is known to activate macrophages [22], also increased levels of inflammatory cytokines. Recent evidence indicates that $4-1 \mathrm{BB}$ signaling results in activation of the MAPK/NF- $\kappa \mathrm{B}$ pathway, which is TNF receptor-associated factor (TRAF)2-dependent [23] in lymphocytes [24]. In adipocytes, we found that stimulation of $4-1 \mathrm{BB}$ activated $\mathrm{p} 38$ MAPK, JNK, IKK and induced $\mathrm{I} \kappa \mathrm{B} \alpha$ protein degradation. On the other hand, stimulation of 4-1BBL led to activation of inflammatory signaling molecules such as Akt and p38 MAPK in macrophages, which is consistent with previous studies $[10,20,21]$. More importantly, we found that treatment with a 4-1BBL neutralizing antibody reduced release of inflammatory cytokines in cocultures at both the mRNA and protein level. These findings together suggest that the 4-1BB/4-1BBL-mediated interaction between adipocytes and macrophages triggers bidirectional inflammatory signaling and is a potent inducer of inflammatory responses in obese adipose tissue (Figure 5).

Interestingly, disruption of the 4-1BB/4-1BBL interaction did not completely suppress release of inflammatory cytokines from cocultured adipocytes and macrophages. This may be due to the presence of other cell surface molecules which participate in cell-cell interactions and mediate inflammatory responses. Indeed, adipocytes and macrophages express many inflammatory receptors and ligands on their surfaces $[6,25,26]$. For example, CD40 and herpes virus entry mediator (HVEM), which are expressed on adipocytes, are considered to be mediators of contactdependent signaling of macrophages, and ablation of these receptors reduces obesity-induced inflammatory responses [26-30]. Thus it is conceivable that other receptors and ligands in addition to $4-1 \mathrm{BB}$ and $4-1 \mathrm{BBL}$ are also involved 
in the interaction between adipocytes and macrophages that leads to the initiation and maintenance of inflammatory responses.

In conclusion, we have demonstrated for the first time that the contact-dependent interaction between adipocytes and macrophages mediated by $4-1 \mathrm{BB}$ and $4-1 \mathrm{BBL}$, which generates bidirectional signals, plays a crucial role in the release of adipose inflammatory cytokines from these cells. 4$1 \mathrm{BB}$ and 4-1BBL, along with other molecules involved in cellcell interaction between adipocytes and macrophages, may be valuable targets for preventing obesity-induced adipose inflammation.

\section{Conflict of Interests}

The authors declare that they have no conflict of interests.

\section{Acknowledgments}

This work was supported by the Midcareer research program through National Research Foundation (NRF) Grant KOSEF (2009-0079485), funded by the Ministry of Education, Science, and Technology (MEST), and the Science Research Center program (Center for Food \& Nutritional Genomics) Grant (2012-0000643) of the NRF of Korea, funded by the MEST.

\section{References}

[1] G. S. Hotamisligil, "Inflammation and metabolic disorders," Nature, vol. 444, no. 7121, pp. 860-867, 2006.

[2] A. Guilherme, J. V. Virbasius, V. Puri, and M. P. Czech, "Adipocyte dysfunctions linking obesity to insulin resistance and type 2 diabetes," Nature Reviews Molecular Cell Biology, vol. 9, no. 5, pp. 367-377, 2008.

[3] L. F. Van Gaal, I. L. Mertens, and C. E. De Block, "Mechanisms linking obesity with cardiovascular disease," Nature, vol. 444, no. 7121, pp. 875-880, 2006.

[4] S. Nishimura, I. Manabe, M. Nagasaki et al., "CD8 ${ }^{+}$effector T cells contribute to macrophage recruitment and adipose tissue inflammation in obesity," Nature Medicine, vol. 15, no. 8, pp. 914-920, 2009.

[5] L. Xie, M. T. Ortega, S. Mora, and S. K. Chapes, "Interactive changes between macrophages and adipocytes," Clinical and Vaccine Immunology, vol. 17, no. 4, pp. 651-659, 2010.

[6] T. Suganami, J. Nishida, and Y. Ogawa, "A paracrine loop between adipocytes and macrophages aggravates inflammatory changes: role of free fatty acids and tumor necrosis factor $\alpha$," Arteriosclerosis, Thrombosis, and Vascular Biology, vol. 25, no. 10, pp. 2062-2068, 2005.

[7] C. N. Lumeng, S. M. Deyoung, and A. R. Saltiel, "Macrophages block insulin action in adipocytes by altering expression of signaling and glucose transport proteins," American Journal of Physiology, vol. 292, no. 1, pp. E166-E174, 2007.

[8] D. Drenkard, F. M. Becke, J. Langstein et al., "CD137 is expressed on blood vessel walls at sites of inflammation and enhances monocyte migratory activity," The FASEB Journal, vol. 21, no. 2, pp. 456-463, 2007.

[9] T. Wen, J. Bukczynski, and T. H. Watts, "4-1BB ligandmediated costimulation of human T cells induces CD4 and
CD8 T cell expansion, cytokine production, and the development of cytolytic effector function," Journal of Immunology, vol. 168, no. 10, pp. 4897-4906, 2002.

[10] Z. Shao and H. Schwarz, "CD137 ligand, a member of the tumor necrosis factor family, regulates immune responses via reverse signal transduction," Journal of Leukocyte Biology, vol. 89, no. 1, pp. 21-29, 2010.

[11] B. Kwon, "CD137-CD137 ligand interactions in inflammation," Immune Network, vol. 9, pp. 84-89, 2009.

[12] S. K. Seo, J. H. Choi, Y. H. Kim et al., "4-1BB-mediated immunotherapy of rheumatoid arthritis," Nature Medicine, vol. 10, pp. 1088-1094, 2004.

[13] T. Haga, J. I. Suzuki, H. Kosuge et al., "Attenuation of experimental autoimmune myocarditis by blocking $\mathrm{T}$ cell activation through 4-1BB pathway," Journal of Molecular and Cellular Cardiology, vol. 46, no. 5, pp. 719-727, 2009.

[14] P. S. Olofsson, L. Å. Söderström, D. Wågsäter et al., “CD137 is expressed in human atherosclerosis and promotes development of plaque inflammation in hypercholesterolemic mice," Circulation, vol. 117, no. 10, pp. 1292-1301, 2008.

[15] H. J. Jeon, J. H. Choi, I. H. Jung et al., "CD137 (4-1BB) deficiency reduces atherosclerosis in hyperlipidemic mice," Circulation, vol. 121, no. 9, pp. 1124-1133, 2010.

[16] H. J. Kim, J. S. Lee, J. D. Kim et al., "Reverse signaling through the costimulatory ligand CD137L in epithelial cells is essential for natural killer cell-mediated acute tissue inflammation," Proceeding of the National Academy of Sciences of the United States of America, vol. 109, pp. E13-E22, 2012.

[17] C. S. Kim, J. G. Kim, B. J. Lee et al., "Deficiency for costimulatory receptor $4-1 \mathrm{BB}$ protects against obesity-induced inflammation and metabolic disorders," Diabetes, vol. 60, pp. 3159-3168, 2011.

[18] W. W. Shuford, K. Klussman, D. D. Tritchler et al., “4$1 \mathrm{BB}$ costimulatory signals preferentially induce $\mathrm{CD} 8^{+} \mathrm{T}$ cell proliferation and lead to the amplification in vivo of cytotoxic T cell responses," Journal of Experimental Medicine, vol. 186, no. 1, pp. 47-55, 1997.

[19] C. S. Kim, T. Kawada, B. S. Kim et al., "Capsaicin exhibits antiinflammatory property by inhibiting IkB-a degradation in LPS-stimulated peritoneal macrophages," Cellular Signalling, vol. 15, no. 3, pp. 299-306, 2003.

[20] L. Söllner, M. M. Shaqireen Kwajah, J. T. Wu, and H. Schwarz, "Signal transduction mechanisms of CD137 ligand in human monocytes," Cellular Signalling, vol. 19, no. 9, pp. 1899-1908, 2007.

[21] D. K. Kim, C. L. Sang, and H. W. Lee, "CD137 ligand-mediated reverse signals increase cell viability and cytokine expression in murine myeloid cells: involvement of mTOR/p70S6 kinase and Akt," European Journal of Immunology, vol. 39, no. 9, pp. 2617-2628, 2009.

[22] H. Schwarz, "Biological activities of reverse signal transduction through CD137 ligand," Journal of Leukocyte Biology, vol. 77, no. 3, pp. 281-286, 2005.

[23] B. Kwon, C. H. Moon, S. Kang, S. K. Seo, and B. S. Kwon, "41BB: still in the midst of darkness," Molecules and Cells, vol. 10, no. 2, pp. 119-126, 2000.

[24] I. Melero, O. Murillo, J. Dubrot, S. Hervás-Stubbs, and J. L. Perez-Gracia, "Multi-layered action mechanisms of CD137 (41BB)-targeted immunotherapies," Trends in Pharmacological Sciences, vol. 29, no. 8, pp. 383-390, 2008.

[25] I. F. Charo and R. M. Ransohoff, "Mechanisms of disease: the many roles of chemokines and chemokine receptors in inflammation," The New England Journal of Medicine, vol. 354, no. 6, pp. 610-621, 2006. 
[26] H. M. Kim, C. S. Jeong, H. S. Choi, T. Kawada, and R. $\mathrm{Yu}$, "LIGHT/TNFSF14 enhances adipose tissue inflammatory responses through its interaction with HVEM," FEBS Letters, vol. 585, no. 3, pp. 579-584, 2011.

[27] M. Croft, W. Duan, H. Choi, S.-Y. Eun, S. Madireddi, and A. Mehta, "TNF superfamily in inflammatory disease: translating basic insights," Trends in Immunology, vol. 33, pp. 144-152, 2011.

[28] H. J. Kim, H. M. Kim, C. S. Kim et al., "HVEM-deficient mice fed a high-fat diet are protected from adipose tissue inflammation and glucose intolerance," FEBS Letters, vol. 585, no. 14, pp. 2285-2290, 2011.

[29] M. Poggi, D. Engel, A. Christ et al., "CD40L deficiency ameliorates adipose tissue inflammation and metabolic manifestations of obesity in mice," Arteriosclerosis, Thrombosis, and Vascular Biology, vol. 31, no. 14, pp. 2251-2260, 2011.

[30] M. Poggi, J. Jager, O. Paulmyer-Lacroix et al., "The inflammatory receptor CD40 is expressed on human adipocytes: contribution to crosstalk between lymphocytes and adipocytes," Diabetologia, vol. 52, no. 6, pp. 1152-1163, 2009. 


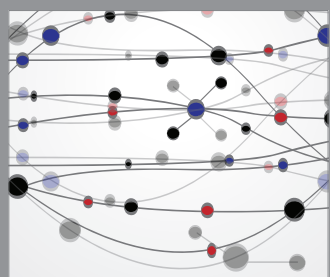

The Scientific World Journal
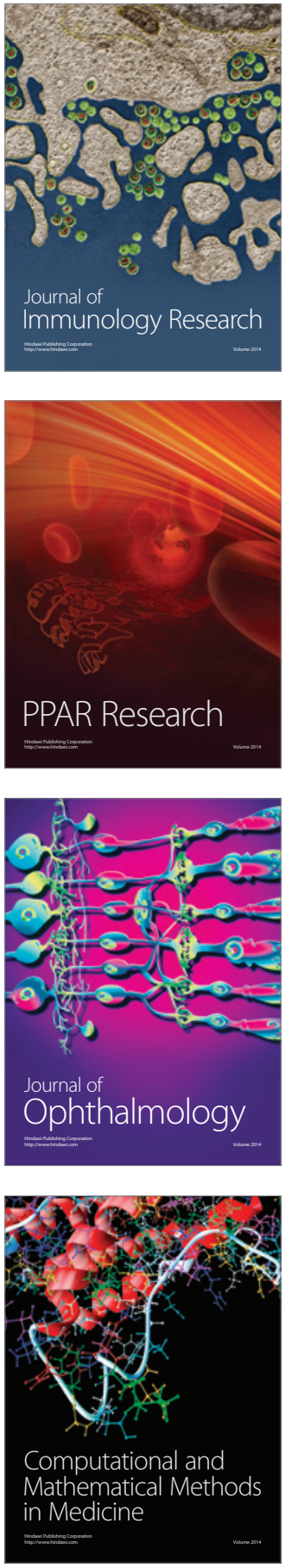

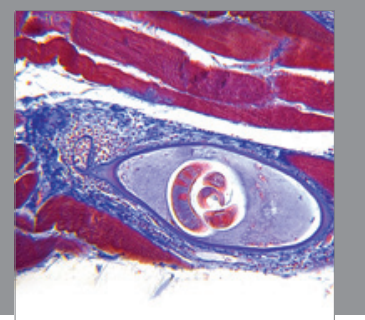

Gastroenterology

Research and Practice
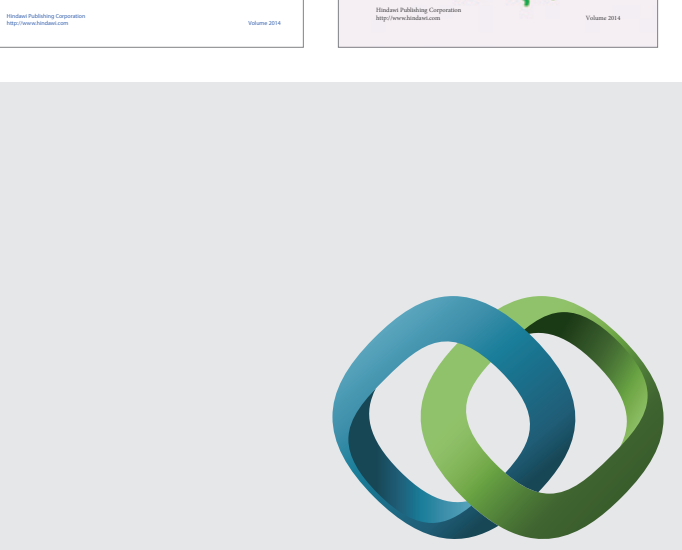

\section{Hindawi}

Submit your manuscripts at

http://www.hindawi.com
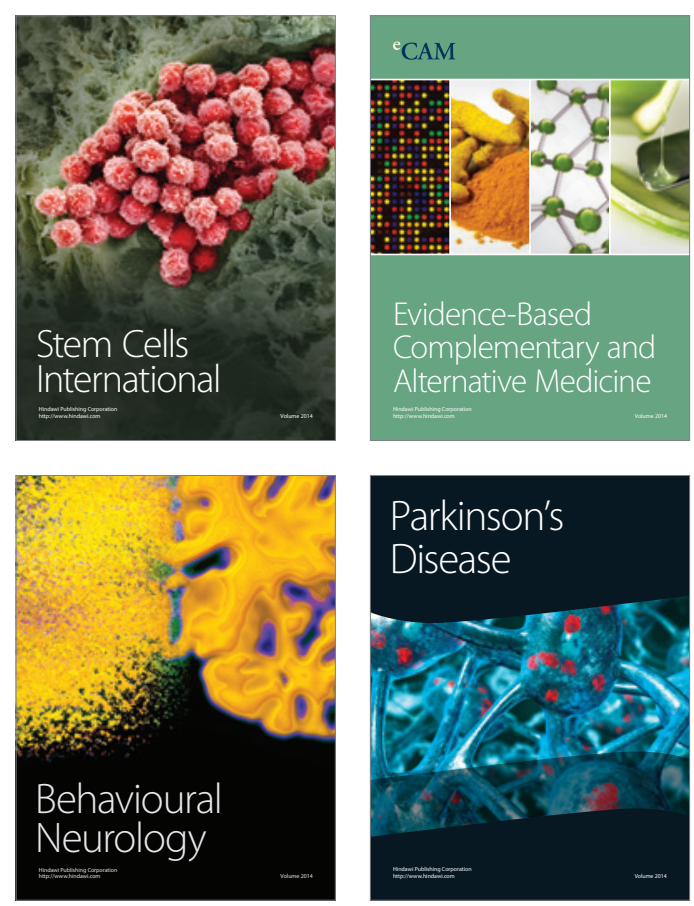

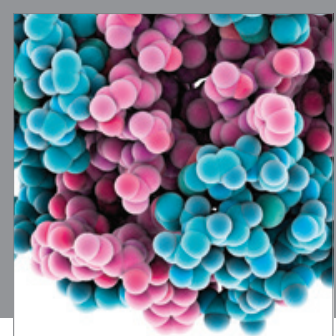

Journal of
Diabetes Research

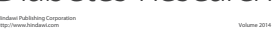

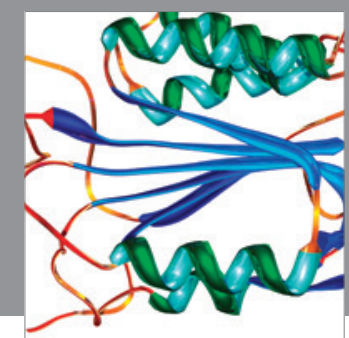

Disease Markers
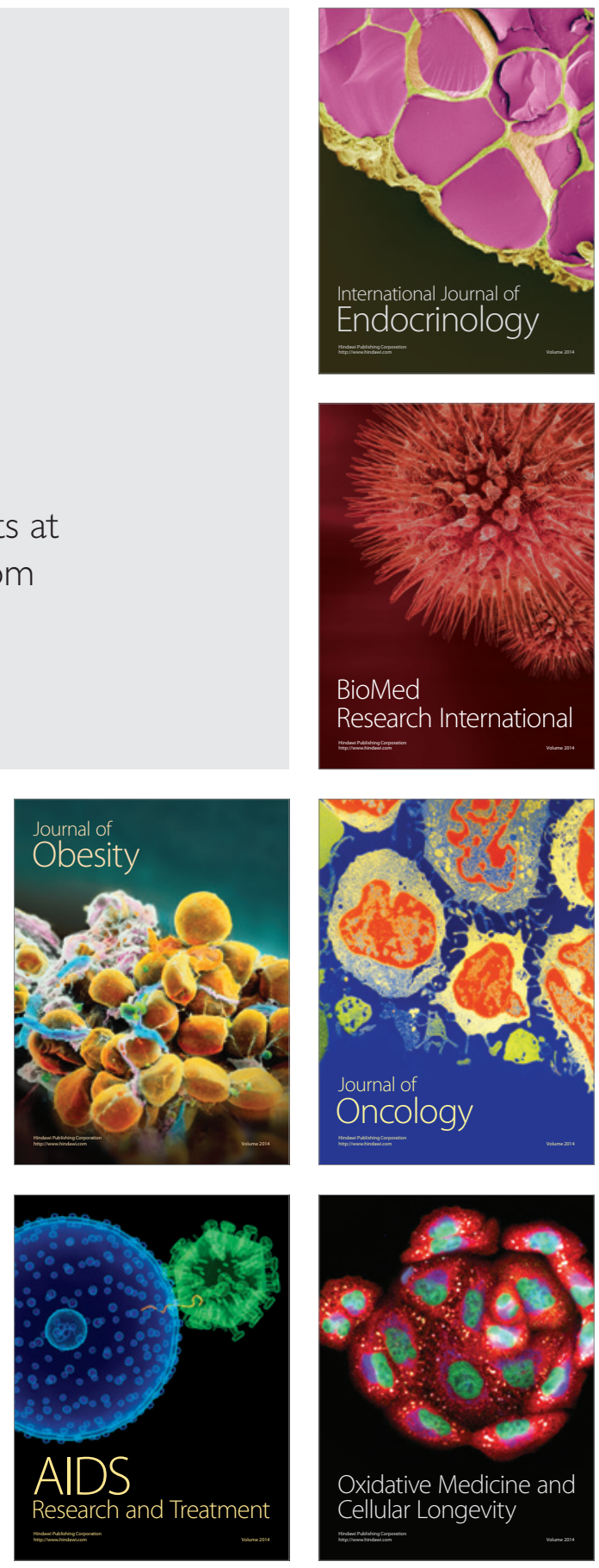